\title{
ESTUDIO COMPARATIVO DE EMPADRE CONTROLADO DE 4 VERSUS 5 PARTOS AL AÑO EN LA CRIANZA DE CUYES
}

\author{
Luis H. Aliaga Rodriguez \\ Universidad Católica Sedes Sapientiae \\ laliaga@ucss.edu.pe
}

Octavio Carhuamaca Rodríguez y Hermenegildo Dorregaray Vilca

Universidad Nacional del Centro del Perú

octavioecr79@hotmail.com

dorre-56@hotmail.com

Resumen: El presente trabajo se desarrolló en los años 2005 y 2006 en la Granja Agropecuaria de Yauris de la U.N.C.P., ubicada en el distrito de El Tambo, provincia de Huancayo, región Junín, a una altitud de 3280 m.s.n.m. El objetivo fue evaluar los parámetros productivos y reproductivos en cuyes sometidos a dos sistemas de empadre (T1 empadre controlado con 4 partos al año versus T2 empadre controlado con 5 partos al año), determinando en ambos tratamientos el número de partos logrados al año, número de crías nacidas al año, número de crías destetadas al año, tamaño promedio de camada al año, número de crías destetadas por cada hembra empadrada en el año y el peso total de crías destetadas por tratamiento en el año, es decir, la productividad ponderal de cada tratamiento expresada por el número de kilos de crías destetadas en el año por cada hembra empadrada.

Los resultados demuestran 25 partos más al año para el tratamiento T2 (empadre controlado con 5 partos al año), diferencia altamente 
significativa para $(\mathrm{p}<0,01)$. Igualmente se registraron 52 más en el tratamiento T2, diferencia significativa para $(\mathrm{p}<0,05)$. En cuanto al tamaño de camada promedio se registró 2,67 crías para el tratamiento T2 (5 partos al año) versus 2,74 crías para el tratamiento T1 (4 partos al año). La diferencia de 0,07 crías a favor del tratamiento T1 resultó, al análisis de varianza, no ser significativa. Respecto al número promedio de crías destetadas al año por parto, se registró 1,29 crías para el tratamiento T2 (5 partos al año) versus 1,23 crías para el tratamiento T1 (4 partos al año). La diferencia de 0,09 crías destetadas por hembra empadrada a favor del tratamiento T2 resultó, al análisis de variancia, no ser significativa. En cuanto al número de crías destetadas al año por hembra empadrada se registró 6,43 crías para el tratamiento T2 (5 partos al año) versus 6,14 crías para el tratamiento T1 (4 partos al año). La diferencia de 0,09 crías destetadas a favor del tratamiento T2 resultó, al análisis de varianza, no ser significativa. Por último se registró el peso total de crías destetadas al año por cada tratamiento de 8179 kilos para el tratamiento T2 (5 partos al año) versus 5763 kilos de crías destetadas para el tratamiento T1 (4 partos al año). La diferencia de 2416 kilos de crías destetadas a favor del tratamiento T2 resulta, al análisis de varianza, ser altamente significativa para $(\mathrm{p}<0,01)$. El trabajo concluye anunciando que es posible aumentar significativamente la productividad de hembras en empadre, expresada en "kilos de destetados por hembra empadrada al año», cuando se programa un empadre controlado de 5 partos al año.

Palabras clave: Empadre, controlado, tratamientos, partos, parámetros productivos, parámetros reproductivos, productividad, destete, crías. 


\section{A COMPARATIVE STUDY OF CONTROLLED BREEDING PAIR OF 4 VERSUS 5 BIRTHS A YEAR IN THE GROWING OF GUINEA PIGS}

ABSTRACT: The present work was carried out in 2005 and 2006, at the Yauris Farm of the U.N.C.P., located in El Tambo district, Huancayo Province in Junín region at an altitude of 3280 meters above sea level.

The objective was to evaluate the productive and reproductive parameters in guinea pigeon undergoing two treatment systems, determining in both the number of successful births per year, the number of babies born per year, the number of weaned babies per year, the average litter size per year, the number of weaned babies for each breeding female per year and the total weight of weaned babies per treatment in the year; that is to say the calculated productivity of each treatment which can be expressed in the number of kilos of weaned babies in the year per every breeding female.

The results show that more than 25 births in a year with treatment T2 (breeding controlled to 5 births per year), with a significant difference $(\mathrm{p}<0,01)$. Likewise, 52 more babies in treatment T2 were registered, with a significant difference $(\mathrm{p}<0,05)$. In relation to the size of the average litter, 2,67 babies resulted from treatment T2 ( 5 births per year) versus 2,74 babies from treatment T1 (4 births per year).

In an analysis of variance, the difference of 0,07 babies in favor of treatment T1 was not statistically significant. With respect to the average number of weaned babies per year per birth, 1,29 babies resulted in treatment T2 ( 5 births per year) versus 1,23 babies in treatment T1 (4 births per year). In an analysis of variance, the difference of 0,09 weaned 
babies per breeding female in favor of treatment T2 (5 births per year) was not statistically significant. With respect to the number of weaned babies per year per breeding female, 6,43 babies resulted from T2 (5 births per year) versus 6,14 babies from treatment T1 ( 4 births per year). In an analysis of variance, the difference of 0,09 weaned babies in favor of treatment T2 was not statistically significant. Finally, the total weight of weaned babies per year resulting from each treatment was 8179 kilos in treatment T2 (5 births per year) versus 5763 kilos in treatment T1 (4 births per year). In an analysis of variance, the difference of 2416 kilos of weaned babies in favor of treatment T2 was highly significant $(\mathrm{p}<0,01)$.

The work concludes stating that it is possible to significantly increase the productivity of breeding females, expressed in «kilos of weaned babies per breeding female per year", when the schedule of breeding is controlled to 5 births per year.

KeYwORDs: controlled breeding, treatment, childbirth, growth performance, reproductive performance, productivity, weaning, pups. 


\section{INTRODUCCIÓN}

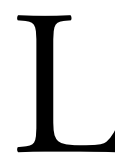

a mayoría de los productores de cuyes utilizan el empadre continuado, en el cual el cuy macho trabaja durante toda su vida reproductiva con año. La importancia del empadre controlado radica en que su programación realizada cada 90 días (4 empadres al año) con una duración de 34 días, de manera que los partos suceden también cada 90 días, durante 34 días; así es posible realizar un mejor control de la parición y por lo tanto una mejor atención a los animales durante esta. El empadre controlado con 4 partos al año es una tecnología generada en la Granja de Yauris de la UNCP (Aliaga 1993: 10) y esta vez se proyecta optimizar este empadre controlado con 5 partos al año, haciéndola de esta forma más eficiente.

La hipótesis planteada en el presente estudio es la siguiente: Un empadre controlado con 5 partos al año, es más eficiente, es decir, más productivo que el empadre controlado de 4 partos al año.

El objetivo del estudio del empadre controlado de 4 versus 5 partos al año fue evaluar los parámetros productivos y reproductivos en ambos tratamientos puestos en estudio, los cuales resumimos en las siguientes variables: (a) El número de partos logrados en el año; (b) el número de crías nacidas en el año; (c) el número de crías destetadas en el año; (d) el tamaño promedio de camada en el año; (e) el número de crías destetadas por cada hembra empadrada en el año; y (f) el peso total de crías destetadas por tratamiento en el año, es decir, la productividad ponderal de cada tratamiento que expresa el número de kilos de crías destetadas en el año por cada hembra empadrada. 


\section{MATERIALES Y MÉTODOS}

- Lugar de ejecución: El estudio se realizó en la Granja Agropecuaria de Yauris de la Universidad Nacional del Centro del Perú. El objetivo fue evaluar los parámetros reproductivos y productivos en ambos tratamientos de manera de determinar la mejor alternativa que permita incrementar la productividad de una explotación de cuyes.

En la investigación se comparó los dos tratamientos de producción de crías de cuyes madres empadradas en forma controlada, la manera de producir 4 versus 5 partos al año.

- Duración: El experimento duró un año

- Población: Se utilizaron animales hembras de tres meses de edad y machos de 4 meses de edad. Las hembras corresponden a las líneas de Yauris, Colorados y Bayos, de tres meses de edad. En cada tratamiento se tomó igual cantidad de hembras de cada línea para evitar la influencia de ellas en el resultado final.

- Tratamientos: Se trata de comparar dos tratamientos de producción de crías de cuyes madres empadradas en forma controlada T1 y T2, de manera de producir 4 versus 5 partos al año. En el tratamiento 1 (4 partos al año) el empadre se efectuó cada 90 días y en el tratamiento 2 (5 partos al año) el empadre se efectuó cada 70 días. -Diseño del experimento: El diseño utilizado fue el Block Randomizado. 


\subsection{VARIABLES DE ESTUDIO}

- Pesos iniciales: Se tomaron los pesos iniciales de las hembras y se randomizó los grupos en cada tratamiento.

- Número de partos al año: Se evaluó y comparó el número de partos habidos al año en cada tratamiento.

- Número de crías nacidas al año: Se determinó el número de crías nacidas durante el año para cada tratamiento.

- Número de crías destetadas al año: Se determinó el número de crías destetadas en el año para cada tratamiento.

- Tamaño de camada promedio: Se determinó el tamaño de camada promedio para cada tratamiento.

- Número de crías destetadas por hembra empadrada por parto: Se determinó el número promedio de crías destetadas por hembra por parto en ambos tratamientos.

- Número de crías destetadas al año por hembra empadrada: Se determinó el número promedio de crías destetadas por hembra empadrada para cada tratamiento.

- Peso total de crías destetadas al año por cada tratamiento: Este parámetro mide la productividad de cada tratamiento expresada en cantidad de kilos destetados por cada tratamiento.

- Alimentación de los animales: Fue el mismo tipo de alimentación para los dos tratamientos.

- Empadre: El empadre se inició el 17 de agosto de 2005. Se formaron 5 bloques experimentales, cada bloque constituido por dos jaulas de empadre. Cada jaula con 6 hembras y un macho, una sometida a 4 empadres al año y la otra a 5 empadres al año. Quince días antes del empadre se les proporcionó a las madres concentrado a discreción 
hasta la finalización del mismo, o sea, durante 49 días. Se espera que esta práctica produzca un «shock» nutritivo en las hembras aumentando su producción hormonal hipofisiaria que se traduce en un incremento en el número de óvulos maduros, o sea, un mayor número de crías, práctica que se conoce por Flushing, demostrada en cuyes (Aliaga 1985).

-Parición: La parición del primer parto en ambos tratamientos se inició la primera semana de octubre de 2005; la parición del segundo parto en ambos tratamientos se inició la primera semana de enero de 2006; la parición del tercer parto en ambos tratamientos se inició la primera semana de mayo de 2006; la parición del cuarto parto en ambos tratamientos se inició la primera semana de agosto de 2006; la parición del quinto parto en el tratamiento 2, se inició la primera semana de noviembre de 2006.

\section{RESULTADOS Y DISCUSIÓN}

Disponiendo de todos los elementos antes mencionados, se determinó proceder con la evaluación. De acuerdo a esto, brindaremos los resultados obtenidos: 
ESTUDIO COMPARATIVO DE EMPADRE CONTROLADO DE 4 VERSUS 5 PARTOS AL AÑO EN LA CRIANZA DE CUYES
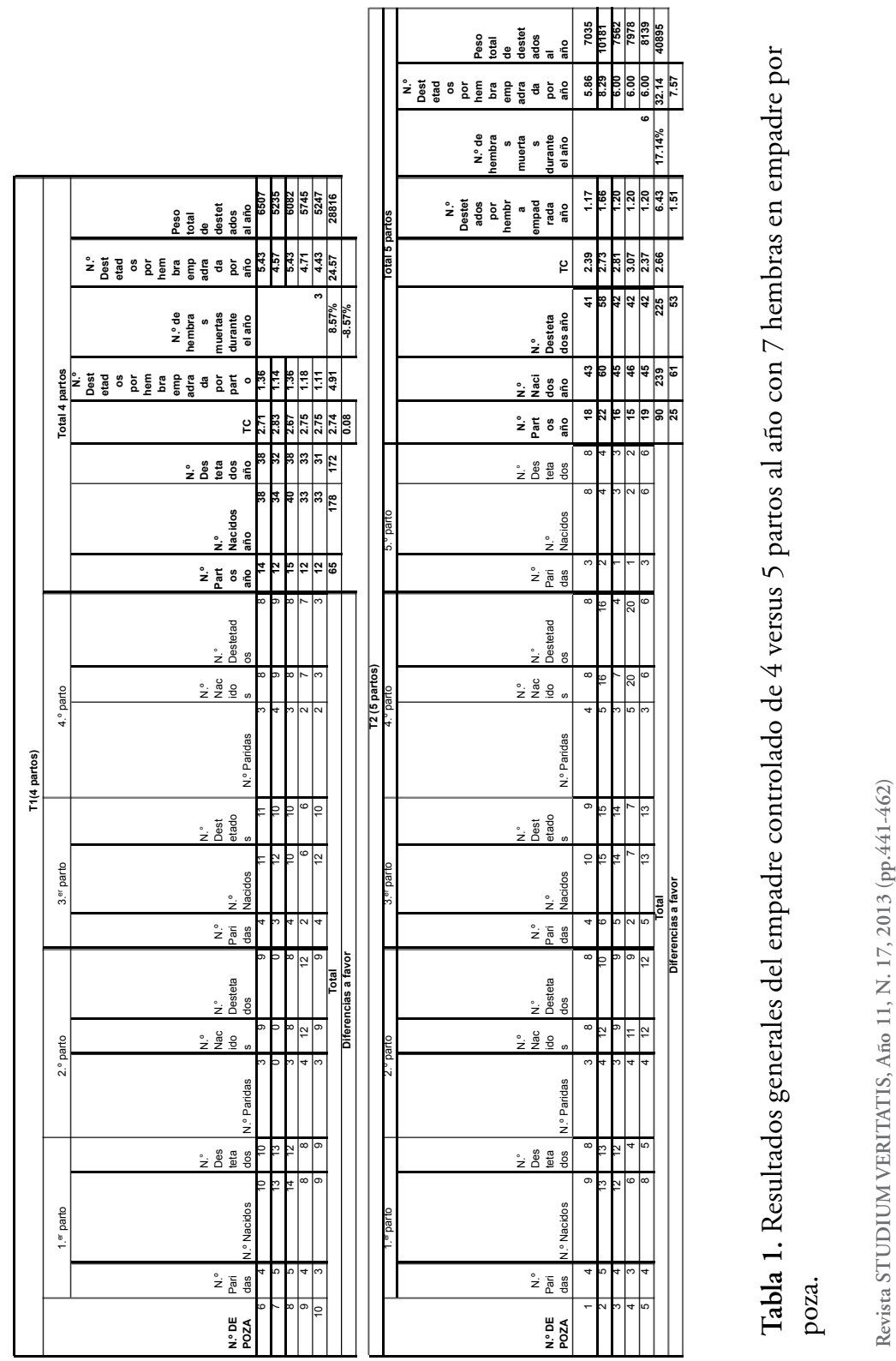


\begin{tabular}{|c|c|c|c|c|}
\hline POZAS & $\overline{T 1}$ & T2 & \multicolumn{2}{|c|}{ TOTAL POZAS } \\
\hline 1 & 14 & 18 & 32 & (a) \\
\hline 2 & 12 & 22 & 34 & (a) \\
\hline 3 & 15 & 16 & 31 & (a) \\
\hline$\overline{4}$ & 12 & 15 & 27 & (a) \\
\hline 5 & 12 & 19 & 31 & (a) \\
\hline TOTAL T & $65 \quad$ (b) & $90 \quad$ (a) & & \\
\hline
\end{tabular}

Letras diferentes:Significación $(p<0.001)$

Tabla 2. N. de partos al año

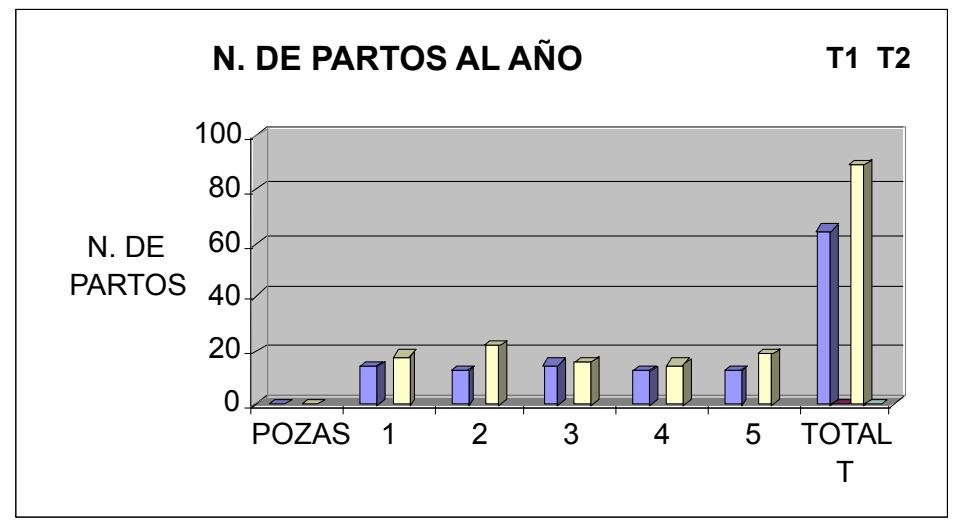

Figura 1. N. de partos al año

Se registraron 90 partos para el tratamiento T2 (5 partos al año) versus 65 partos para el tratamiento T1 (4 partos al año). La diferencia de 25 partos más a favor del tratamiento T2, resulta al análisis de varianza, altamente significativa para $(\mathrm{p}<0,01)$.

La diferencia es debida exclusivamente al mayor número de partos durante el año (5 versus 4). Trabajos en reproducción de cuyes con empadre intensivo o continuo versus empadre semiintensivo realizados por A.J.H Tomlinsos demuestran este hecho (Aliaga 1979: 74-80). 


\begin{tabular}{|c|c|c|c|c|}
\hline POZAS & T1 & T2 & \multicolumn{2}{|c|}{ TOTAL POZAS } \\
\hline 1 & 38 & 43 & 81 & (a) \\
\hline 2 & 34 & 60 & 94 & (a) \\
\hline 3 & 40 & 45 & 85 & (a) \\
\hline 4 & 33 & 46 & 79 & (a) \\
\hline 5 & 33 & 45 & 78 & (a) \\
\hline TOTAL T & 178 & (b) & $239 \quad$ (a) & \multicolumn{1}{|c}{} \\
\cline { 1 - 3 } & & & & \multicolumn{2}{|c|}{}
\end{tabular}

Letras diferentes:Significación $(p<0.001)$

Tabla 3. Número de crías nacidas al año

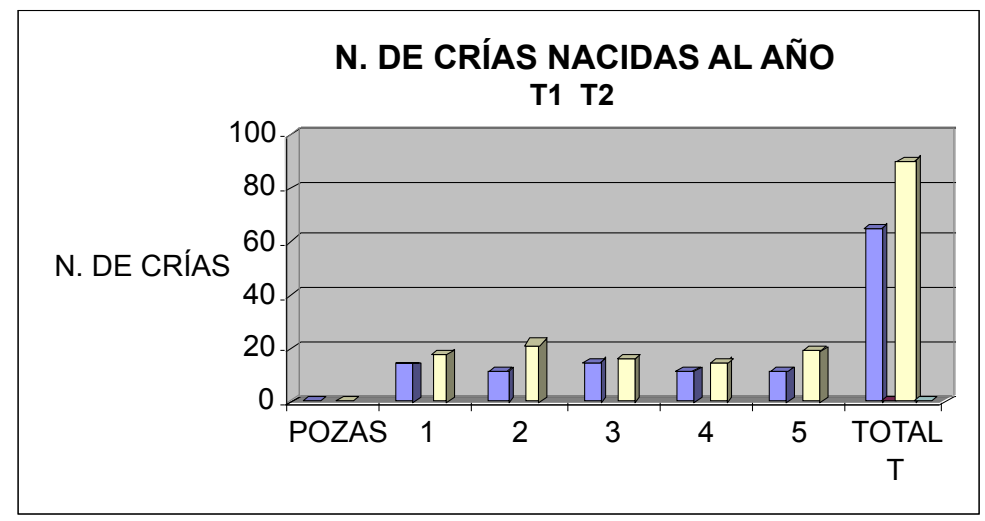

Figura 2. Número de crías nacidas al año.

Se registraron 239 crías nacidas al año para el tratamiento T2 (5 partos al año) versus 178 para el tratamiento T1 (4 partos al año). La diferencia de 61 crías nacidas al año a favor del tratamiento T2 resulta, al análisis de varianza, altamente significativa para $(\mathrm{p}<0,01)$.

La diferencia de 61 crías a favor del tratamiento T2 se debe exclusivamente al mayor número de partos habidos en el año, demostrado también por A.J.H Tomlinson (Aliaga 1979: 71-73). 


\begin{tabular}{|c|c|c|c|c||c|}
\hline POZAS & T1 & T2 & \multicolumn{2}{|c|}{ TOTAL POZAS } \\
\hline 1 & 38 & 41 & 79 & (a) \\
\hline 2 & 32 & 58 & 90 & (a) \\
\hline 3 & 38 & 42 & 80 & (a) \\
\hline \hline 4 & 33 & 42 & 75 & (a) \\
\hline 5 & 31 & 42 & 73 & (a) \\
\hline TOTAL T & 172 & (b) & 225 & (a) & \multicolumn{2}{|c}{} \\
\cline { 1 - 5 }
\end{tabular}

Letras diferentes:Significación $(p<0.005)$

Tabla 4. Número de crías destetadas al año

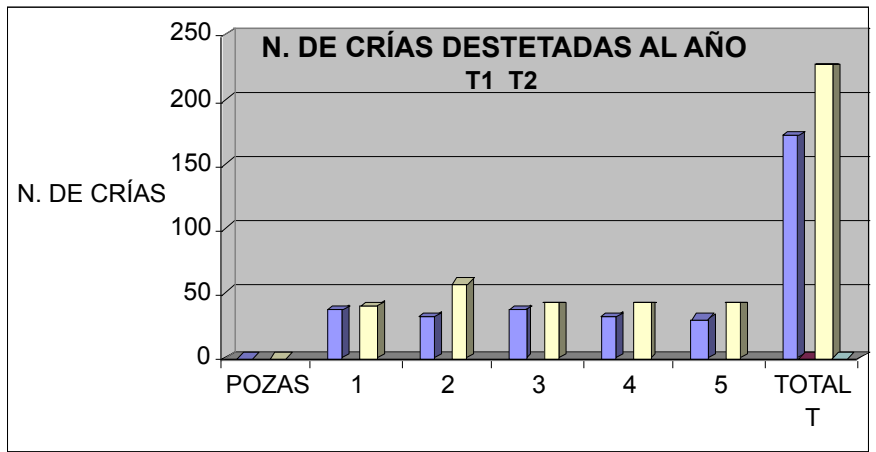

Figura 3. N. de crías destetadas al año

Se registraron 225 crías destetadas al año para el tratamiento T2 (5 partos al año) versus 172 para el tratamiento T1 (4 partos al año). La diferencia de 53 destetadas al año a favor del tratamiento T2 resulta, al análisis de varianza, significativa para $(\mathrm{p}<0,05)$.

La diferencia de 53 crías destetadas a favor del tratamiento T2, se debe al mayor número de partos habidos en este tratamiento, así lo demuestra también Tomlinson en su trabajo de investigación en reproducción de cuyes (Aliaga 1979: 73). 


\begin{tabular}{|c|l|l|c|l|}
\hline POZAS & T1 & T2 & TOTAL POZAS \\
\hline 1 & 2.71 & 2.39 & 5.10 & (a) \\
\hline 2 & 2.83 & 2.73 & 5.56 & (a) \\
\hline 3 & 2.67 & 2.81 & 5.48 & (a) \\
\hline 4 & 2.75 & 3.07 & 5.82 & (a) \\
\hline \hline 5 & 2.75 & 2.37 & 5.12 & (a) \\
\hline TOTAL Ts & $2.74 \quad$ (a) & $2.67 \quad$ (a) & \multicolumn{1}{l}{} \\
Tetras diferentes:Significación (p<0.005)
\end{tabular}

Tabla 5. Tamaño de camada promedio al año

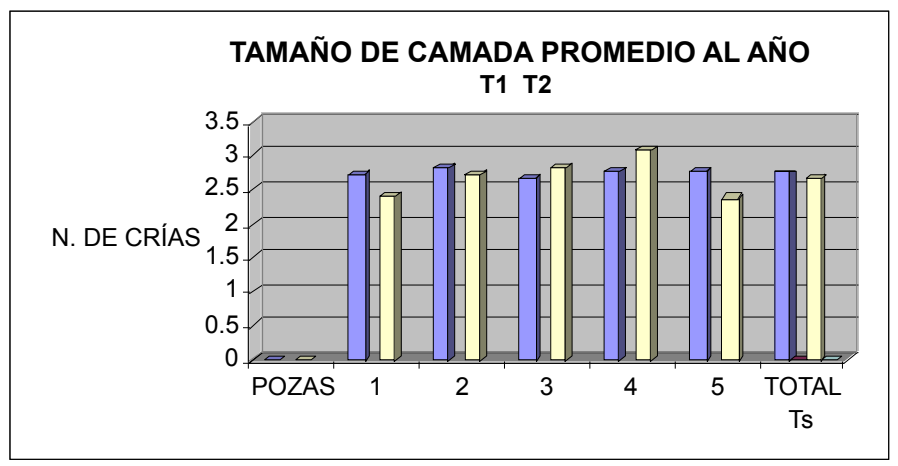

Figura 4. Tamaño de camada promedio al año

Se registró un tamaño de camada promedio de 2,67 crías para el tratamiento T2 (5 partos al año) versus 2,74 crías para el tratamiento T1 (4 partos al año). La diferencia de 0,07 crías a favor del tratamiento T1 no resulta ser, al análisis de varianza, significativa.

El tamaño de camada es un parámetro que depende de la buena alimentación y de la calidad genética del individuo. Por esta razón, en el ensayo presente la diferencia habida en este parámetro no es significativa (Aliaga 1979: 58). 


\begin{tabular}{|c|c|c|c|c|}
\hline POZAS & T1 & T2 & \multicolumn{2}{|c|}{ TOTAL POZAS } \\
\hline 1 & 1.36 & 1.17 & 2.53 & (a) \\
\hline 2 & 1.14 & 1.66 & 2.80 & (a) \\
\hline$\overline{3}$ & 1.36 & 1.20 & 2.56 & (a) \\
\hline$\overline{4}$ & $\overline{1.18}$ & 1.20 & 2.38 & (a) \\
\hline 5 & 1.11 & 1.20 & 2.31 & (a) \\
\hline TOTAL Ts & $1.23 \quad$ (a) & $1.29 \quad$ (a) & & \\
\hline
\end{tabular}

Letras diferentes:Significación $(p<0.005)$

Tabla 6. Número promedio de crías destetadas por parto por hembra empadrada al año

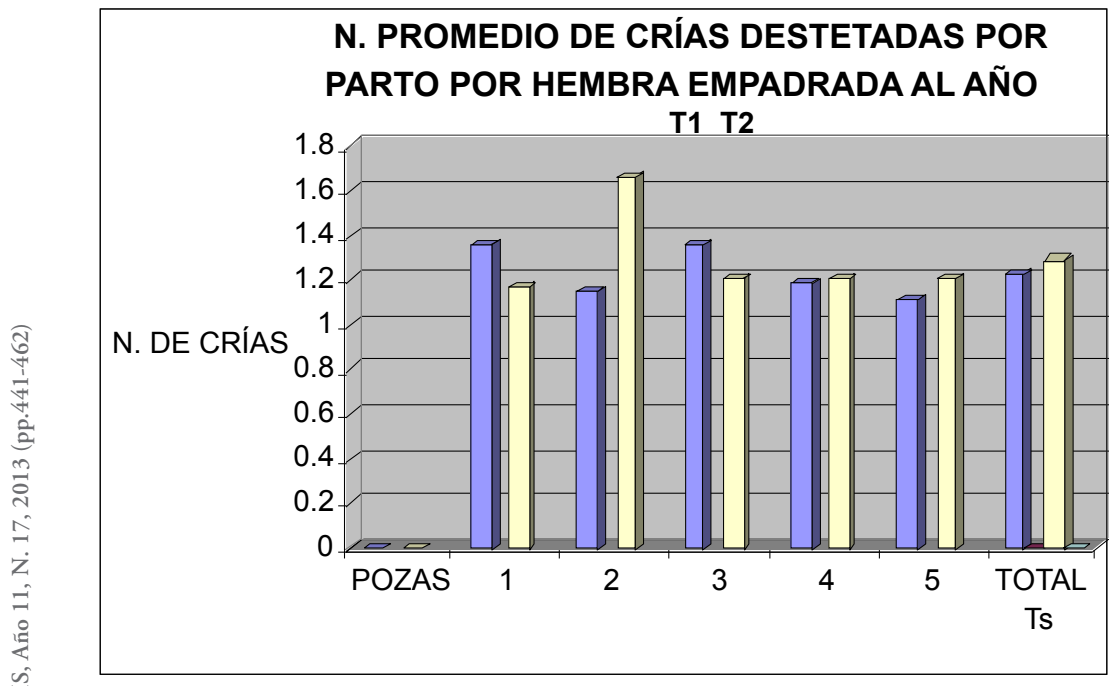

Figura 5. N. Promedio de crías destetadas por parto por hembra empadrada al año

Se registró un número promedio de crías destetadas al año por parto de 1,29 crías para el tratamiento T2 (5 partos al año) versus 1,23 crías para el tratamiento T1 (4 partos al año). La diferencia de 0,09 crías destetadas 
por hembra empadrada a favor del tratamiento T2 resultó no ser, al análisis de varianza, significativa.

El número de crías destetadas al año por parto es un factor ligado al tamaño de camada (Aliaga 1979: 58) y como este último es un factor que depende más de la alimentación (Aliaga 1995: 47) y de la calidad genética del individuo, la diferencia en crías destetadas al año por parto no resulta significativa.

\begin{tabular}{|c|c|c|c|c|}
\hline POZAS & T1 & T2 & \multicolumn{2}{|c|}{ TOTAL POZAS } \\
\hline 1 & 5.43 & 5.86 & 11.29 & (a) \\
\hline 2 & 4.57 & 8.29 & 12.86 & (a) \\
\hline 3 & 5.43 & 6.00 & 11.43 & (a) \\
\hline 4 & 4.71 & 6.00 & 10.71 & (a) \\
\hline 5 & 4.43 & 6.00 & 10.43 & (a) \\
\hline TOTAL Ts & $4.91 \quad$ (b) & $6.43 \quad$ (a) & \multicolumn{2}{|c}{} \\
\hline
\end{tabular}

Letras diferentes:Significación $(p<0.005)$

Tabla 7. Número total de crías destetadas por parto por hembra empadrada al año.

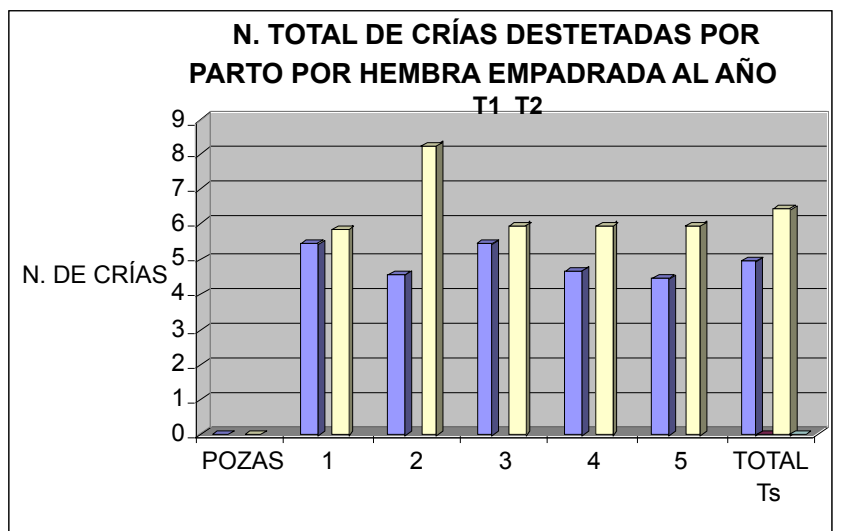

Figura 6. N. total de crías destetadas por parto por hembra empadrada al año 
Se registró un número de crías destetadas al año por hembra empadrada de 6,43 crías para el tratamiento T2 (5 partos al año) versus 4,91 crías para el tratamiento T1 (4 partos al año). La diferencia de 1,52 crías destetadas a favor del tratamiento T2 resultó ser, al análisis de varianza, significativa para $(\mathrm{p}<0,05)$.

La diferencia a favor del tratamiento T2 (empadre controlado con 5 partos al año) se debe al mayor número de partos al año (Aliaga 1979: 73).

\begin{tabular}{|c|c|c|c|c|}
\hline POZAS & T1 & T2 & \multicolumn{2}{|c|}{ TOTAL POZAS } \\
\hline 1 & 6.507 & 7.035 & 13.542 & (a) \\
\hline 2 & 5.235 & 10.181 & 15.416 & (a) \\
\hline 3 & 6.082 & 7.562 & 13.644 & (a) \\
\hline 4 & 5.745 & 7.978 & 13.723 & (a) \\
\hline 5 & 5.247 & 8.139 & 13.386 & (a) \\
\hline TOTAL Ts & 5.763 (b) & 8.179 (a) & & \\
\hline
\end{tabular}

Letras diferentes:Significación $(p<0.001)$

Tabla 8. Peso total de crías destetadas en kg por hembra destetadas al año (productividad anual) 


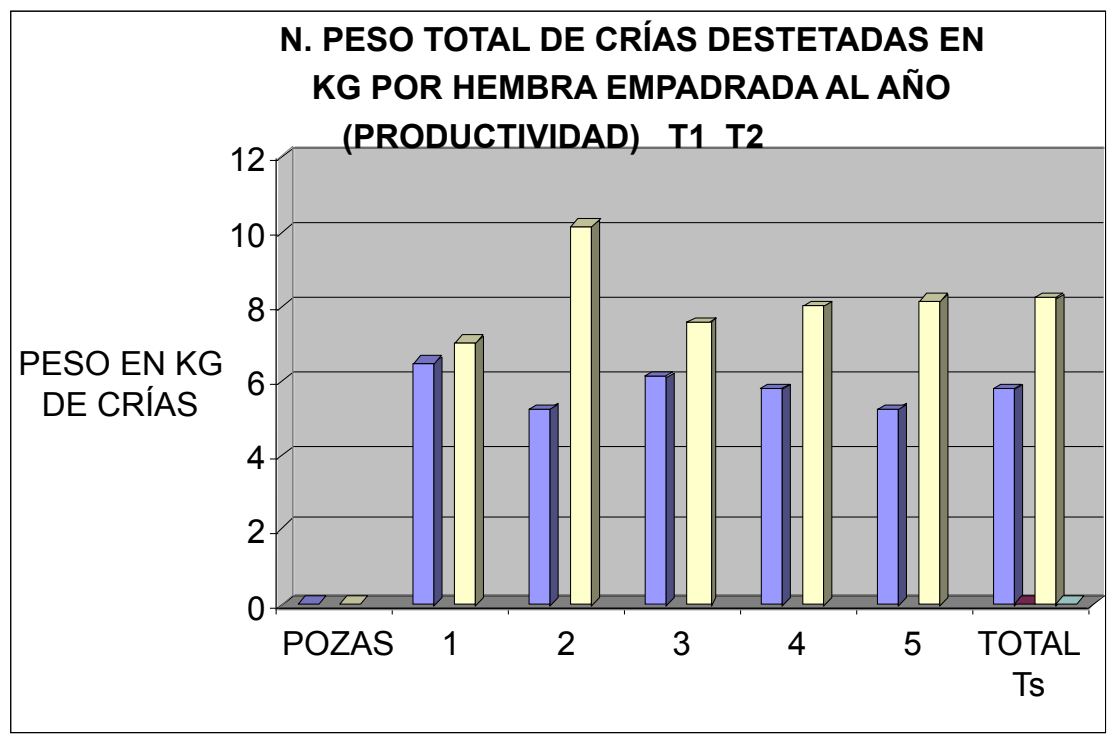

Figura 7. N. Peso total de crías destetadas en kg por hembra empadrada al año

Se registró el peso total de crías destetadas al año por cada tratamiento de 8179 kilos para el tratamiento T2 (5 partos al año) versus 5763 kilos de crías destetadas para el tratamiento T1 (4 partos al año). La diferencia de 2416 kilos de crías destetadas a favor del tratamiento T2 resultó ser, al análisis de varianza, altamente significativa para $(\mathrm{p}<0,001)$.

Este último resultado concentra todos los parámetros anteriormente evaluados y mide la productividad ponderal de ambos tratamientos, es decir, la cantidad de kilos destetados al año por cada hembra empadrada.

$\mathrm{Al}$ existir mayor número de partos al año en el tratamiento T2, hubo también mayor cantidad de crías destetadas por hembra empadrada y 
por lo tanto, mayor peso de crías destetadas por cada hembra empadrada al año (Aliaga 1979: 75 y Aliaga 1995: 55).

\section{CONCLUSIONES}

- Los resultados demuestran 25 partos más al año para el tratamiento T2 (empadre controlado con 5 partos al año), diferencia altamente significativa para $(\mathrm{p}<0,01)$.

-Se registraron 52 crías destetadas más, en el tratamiento T2, diferencia significativa para $(\mathrm{p}<0,05)$.

-En cuanto al tamaño de camada promedio se registró 2,67 crías para el tratamiento T2 (5 partos al año) versus 2,74 crías para el tratamiento T1 (4 partos al año). La diferencia de 0,07 crías a favor del tratamiento T1 resultó, al análisis de varianza, no ser significativa.

- Respecto al número promedio de crías destetadas al año por parto se registró 1,29 crías para el tratamiento T2 (5 partos al año) versus 1,23 crías para el tratamiento T1 (4 partos al año). La diferencia de 0.09 crías destetadas por hembra empadrada a favor del tratamiento T2 resultó, al análisis de varianza, no ser significativa.

-En cuanto al número de crías destetadas al año por hembra empadrada se registró 6,43 crías para el tratamiento T2 (5 partos al año) versus 6,14 crías para el tratamiento T1 (4 partos al año). La diferencia de 0,09 crías destetadas a favor del tratamiento T2 resultó, al análisis de varianza, no ser significativa.

- Respecto al peso total de crías destetadas al año por cada tratamiento de 8179 kilos para el tratamiento T2 (5 partos al año) versus 5763 kilos de crías destetadas para el tratamiento T1 (4 partos al año). La 
diferencia de 2416 kilos de crías destetadas a favor del tratamiento T2 resulta, al análisis de varianza, ser altamente significativa para $(\mathrm{p}<0,01)$.

-Es posible aumentar significativamente la productividad de hembras en empadre, expresada en «kilos de destetados por hembra empadrada al año", cuando se programa un empadre controlado de 5 partos al año. 
*Luis Aliaga Rodríguez es ingeniero agrónomo por la Universidad Nacional Agraria La Molina. Es autor del libro Producción de cuyes y de varios manuales sobre la selección, mejora y crianza de cuyes. Es Gerente General de la Agropecuaria Inka Cuy S.A.C., empresa peruana dedicada a la explotación de cuyes con fines de producción de reproductores de calidad, la cual fue premiada con el título «Mejor criador de cuyes» en la X Feria de Ganadería La Molina en el 2006. Es consultor y asesor técnico de empresas ligadas a la crianza de cobayos. Es profesor emérito de la Universidad Nacional del Centro del Perú. Actualmente, es Vicerrector Académico de la Universidad Católica Sedes Sapientiae. En el año 2013 fue investido Doctor Honoris Causa por la Universidad Nacional del Centro del Perú (UNCP), en reconocimiento a su trayectoria y sus aportes en el campo de la zootecnia como investigador y docente en importantes universidades de nuestro país. *Octavio Carhuamaca Rodríguez es doctor en Educación y postdoctorado en Medio Ambiente y Desarrollo Sostenible por la Universidad Inca Garcilaso de la Vega. Es Ingeniero Zootecnista por la Universidad Nacional del Centro del Perú. Ha realizado una segunda especialidad en Didáctica Universitaria por la Universidad Peruana Los Andes. Asimismo, ha llevado a cabo diversas pasantías en países de América y Europa. Se ha desempeñado como docente y directivo de universidades a nivel de Pregrado y Postgrado por más de 20 años. Ha laborado en empresas ganaderas de la zona Alto Andina. Actualmente es director de Sistema Administrativo activo en la Universidad Nacional del Centro del Perú. Ha realizado trabajos de investigación científica en cultivo y manejo de pasturas en la Universidad Nacional Agraria la Molina. Es integrante del equipo de investigadores liderados por el conspicuo profesional Dr. Luis Aliaga Rodríguez en la formación de la primera raza de cuyes en el mundo, «Raza Wanka», realizada 
en la Granja Agropecuaria de Yauris de la Universidad Nacional del Centro del Perú, en 1992.

${ }^{*}$ Hermenegildo Dorregaray Vilca es bachiller en Zootecnia. Ha participado en exposiciones nacionales y ferias agropecuarias por más de 15 años. Ha laborado en el Centro Latinoamericano de Especies Menores CLEMTULUA-COLOMBIA. Además, ha participado en el programa especial en producción animal IPC LIVESTOCK BARNEVELD COLLEGEHOLANDA. Ha realizado trabajos de investigación en animales menores en la Granja Agropecuaria de Yauris de la Universidad Nacional del Centro del Perú. Asimismo, participó en la obtención de la «Raza Wanka» de cuyes de esta institución. Actualmente, se desempeña como administrativo de la Universidad Nacional del Centro del Perú. 


\section{BIBLIOGRAFÍA}

Aliaga Rodríguez, Luis

1979

Producción de cuyes. Huancayo: Departamento de impresiones de la Universidad Nacional del Centro del Perú.

1985 «Sistema de Empadre Controlado con Flushing proteico en Cuyes». Ponencia presentada en la viII Reunión Científica Anual de la Asociación Peruana de Producción Animal APPA. Lima, 12 al 16 de noviembre de 1985.

1993

Manual técnico sobre crianza de cuyes. Lima: Instituto Nacional de Investigación Agraria (INIA).

1995

«Reproducción, Sistemas de Empadre en Cuyes». En Crianza de Cuyes. Serie Guía Didáctica N. R1-95. Lima: Instituto Nacional de Investigación Agraria (INIA). 